\title{
Automated Unsupervised Geomorphometric Classification of Earth Surface for Landslide Susceptibility Assessment
}

\author{
Maria Ioannilli and Alessandro Paregiani \\ University of Rome "Tor Vergata",Via del Politecnico, 1 - 00133 Rome - Italy \\ ioannill@ing.uniroma2.it, a.paregiani@gmail.com
}

\begin{abstract}
The aim of this work is to define an automated method of terrain classification in order to evaluate the correlation degree between topographic forms of the analyzed territory and registered landslide phenomena with a Landslide Inventory and DEMs as unique input data. A reliable procedure that identifies areas subject to different levels of susceptibility by a geomorphometric approach is presented. The main objective is reached by means of intermediate steps. The first step is the individuation of a set of measures, a geometric signature, that describes topographic form to distinguish among geomorphically different landscapes; the identified parameters are slope gradient, aspect, plan and section curvatures, local convexity and surface texture, computed from a 30x30m square-grid digital elevation model (DEM). The second step is the classification of the analyzed territory in eleven classes using the geometric signature tool. Finally, the eleven classes are statistically correlated with the Landslide Inventory of the analyzed territory. This work represents a useful tool in large-scale landslide susceptibility analysis. In fact, the application of this repeatable and reliable procedure may return the best results in a short time and with low economic resources, providing specific useful information in planning Civil Protection investigations and operations.
\end{abstract}

Keywords: Terrain Classification, Geomorphometry, Landslide, Susceptibility, Hazard Analysis, Spatial Analysis.

\section{Introduction}

There are no universally accepted forecasting methods of "natural hazard" and in particular of landslide hazard. The proliferation of studies on landslide phenomena demonstrates the increasing interest in this field. These studies aim to understand the basic mechanisms that regulate these phenomena, in order to develop interpretive models for hazard assessment.

In the literature there are different methodological approaches. From a Civil Protection planning perspective, and in particular with forecasting and preventing purposes, the study of landslide phenomena does not end with the investigation of a single event; on the contrary, its first objective is an understanding of the potential behaviour over a wide territory, and subsequently an assessment of the hazard existing on an extended territorial 
domain and not only on a specific mountainside surface. A large-scale investigation allows to consider and analyze the territory in its entirety and then to orientate local specific analysis where the proclivity to a catastrophic event is the highest registered.

A landslide susceptibility analysis has as its aim the evaluation of the different proclivity levels of a territory. Susceptibility analysts have to consider and to analyze different factors and their temporal evolution in order to reach this objective. These factors represent the shapes (morphology) or the specific physical characteristics (material, consistency, structure) of a territory.

Despite the various existing methodological approaches, all analyzed methods are based on a conceptual model, which consists of mapping landslides in a given region (target) or part of it (training area) and of the idea that it is more probable that a landslide will occur in the future where it has already occurred in the past, under certain instability conditions.

There are direct and indirect methods to build up a model and then to use it in the assessment of landslide susceptibility. The statistical method is certainly one of the most frequently used in landslide susceptibility analysis [1]. A variable number of morphological and geological (such as lithology, land cover, pedology) factors are set and correlated with the landslide distribution, in order to measure the correlation degree of each factor with landslides and therefore the relative weights to combine the relevant factors in a susceptibility map. The objectivity of the first phase of analysis (to determine the relevant factors and how they affect the instability) is the main positive feature, whereas the negative aspect is the lack of a correlation analysis between the various factors. In heuristic deductive method, the analyst's knowledge of instability factors is fundamental for a well-made analysis. In fact, instability factors are a-priori selected, classified and weighed, not considering the distribution of events [1]. After the definition of these parameters and their weights, susceptibility map ensues from their combination. At this point the map is compared with landslide inventory to verify the accuracy of the original hypothesis. This method, unlike the statistical one, considers the existing interactions among instability factors, but it is affected by a high subjectivity.

The geomorphometric approach eliminates the negative aspects of the two mentioned methods, referring to parameters representing the shape of the territory. It is a direct approach that removes the subjectivity of the heuristic one; running a clustering classification by introducing the concept of "geometric signature", it identifies combinations of parameters, and combinations that are more destabilising for a certain territory.

In order to define geomorphometric classes and to analyze landslide susceptibility, a terrain-unit choice is required. The issue of defining a feasible terrain-unit is common to many other different types of investigations ranging from pollution control to archaeological surveys. A suitable terrain-unit is necessary in order to generate a database of the environmental data needed to describe a given process or phenomenon, and to build up a predictive model of the spatial distribution of the phenomenon.

\section{Geomorphometry}

Geomorphometry is the science of quantitative land-surface analysis. It draws upon mathematical, statistical and image-processing techniques to quantify the shape of the earth topography at various spatial scales. 
The quantitative analysis of a territory, and in particular of its shape, eliminates the limitations of the qualitative topographic information. It is important to note how the characterization of the topography tends to be qualitative and subjective, because the nomenclature is verbal rather than technical. Adjectives such as hilly, steep, mountainous and rough are used with different meanings by different observers, according to their personal experience and the scale of observation. A consequence of this subjectivity is that territories, even very different ones, can be encoded with identical terms. The different meanings of these qualitative terms make the communication about topographical information difficult.

The study of the geomorphometry stems from the need to establish a reliable numerical model in order to describe the earth shape. The quantitative characterization of topographical shape is a multidisciplinary technique applicable at any scale of analysis . Therefore, the measure of earth shape is independent of the scale and type of surface.

\subsection{The Geometric Signature}

One analytic tool of numerical land-surface classification is the geometric signature.

In [2] the signature was defined as "a set of measurements sufficient to identify unambiguously an object or a set of objects". In [3], this concept was adapted from remote sensing to geomorphology, defining the geometric signature as "a set of measurements that describes topographic form well enough to distinguish geomorphologically disparate landscapes".

Natural surface processes create different forms that are recognizable in the field. The geometric signature abstracts those forms from contour maps or from DEMs and expresses them numerically. The measures that constitute a geometric signature express different but complementary attributes of surface form [4]. The optimal set of elements defining a signature has been subject of debate and remain a variable depending on the specific problem at hand and the spatial scale.

\section{Classification Method}

The definition of a reliable and semi-automated method applicable to landslide susceptibility analysis is organized and computed in three different phases: in the first phase, existing methodologies are applied to the analyzed territory and the obtained results are compared; in the second one, a new methodology is defined and experimented in order to improve the obtained classifications; finally, a statistical correlation analysis between the Landslide Inventory and the obtained classification is implemented.

The analysis is developed on the territory of the Province of Rome.

One of the first and most sensitive operations in a landslide susceptibility analysis is the choice of the terrain-unit, because it could affect noticeably the results of analysis. It is worth discussing the criteria for selecting the terrain-unit, as a portion of land surface which contains a set of ground conditions which differ from the adjacent units across definable boundaries [5]. It depends on various parameters, such as the input data type, the scale of analysis, the desired output data quality and spatial resolution and on the availability of analytic and information tools [1]. 
In Table 1 different types of terrain-unit are reported. In the literature, various methods have been proposed in order to define the terrain-unit.

In natural environments, the interrelations between materials, forms and processes result in morphological boundaries which frequently reflect geomorphological and geological differences. The geomorphologic unit was applied in many environmental analysis, but its main drawback lies in the fact that different investigators often classify a region in a different way, therefore the technique is intrinsically subjective. A second approach implies the division of the region into grid-cells of given size which become the terrain-unit. Grid-cells are particularly preferred in the representation of continuous variables, such as slope gradient, altitude and aspect. One of the most relevant advantages of this method is the computational simplicity and velocity that an array offers, while they are characterized by a low physical meaning. The third method implies the classification of each slope-instability factor into a few significant classes which will be stored into a single map or layer. By overlaying all the layers, homogeneous domains are defined. Although the technique seems to be fully objective, its main weakness refers to the subjectivity in factor classification. Moreover, by overlaying more than 4 maps, thousands of small, statistically meaningless, domains can be generated. The fourth approach represented in Table 1, based on the partition of a region into slope-units or sub-basin, seems the most appropriate for landslide hazard assessment, but it has a substantial limitation in manually identifying slope-units or sub-basin boundaries [1].

Table 1. Comparison among Different Terrain-Units for Landslide Hazard Mapping

\begin{tabular}{|l|l|l|l|}
\hline Terrain-Unit & Consistency & Physical Meaning & Technique \\
\hline Geomorphologic Unit & Low & High & Manual \\
\hline Grid-Cell & High & Low & Automatic \\
\hline Unique Condition Unit & High & Medium & Automatic \\
\hline Slope Unit & High & Medium & Automatic \\
\hline
\end{tabular}

In this study, grid-cells are used as terrain-unit.

The starting-point data in the computation of the different morphometric parameters is a 30x30m DEM, computed by interpolating the altitude-points extracted from contour lines (10m interval) of the Technical Regional Cartography of Lazio, while the Landslide Inventory of Tevere Basin Authority (PAI), converted in grid format, is used in the correlation analysis. The Landslide Inventory differentiates seven types of phenomena and the number of events totally registered on the analyzed territory is 351 with a total area of 19,35 square kilometres. The total area of the Province of Rome is about 5.352 square kilometres.

The computational tool is ARC/INFO and the analysis are carried out in a semiautomated way through ARC/INFO procedures.

\subsection{Applying State-of-the-Art Classification Methods}

Analyzing the state-of-the-art, there is a wide range of algorithmic approaches to earth surface classification. Some classifications are supervised, and therefore types of 
topography are recognized starting from selected "training samples", while others are unsupervised, unconstrained by pre-set conditions, and allow the input data themselves to determine "optimal" categories.

Thus, the first phase is the application of three previous unsupervised studies ([6], [7] and [8]) about the evaluation of geometric signature. In these studies single-cell and multi-focal parameters are computed and analyzed. In a single-cell analysis, the edge of the cell has no physical relationship with the neighborhood environmental parameters and therefore the process and the statistical analysis are quick and simple. In the literature there are many cases of application of this type of analysis. This method identifies the morphometric features scanning the DEM with a local window (3x3) and examines the relationships of the cell with its processed neighbourhood [9]. The use of a window in the calculation of parameters is due to the lack of meaning of a part of them, such as the slope gradient, if they are not in physical relation to a context. The term "single-cell" is due to the fact that the outcome of the analysis leads to the determination of "unique" value of the cell. Strictu sensu, the altitude is the only real single-cell parameter among the considered ones in these studies, as reported in Table 2.

In computing multi-focal parameters, a local variable window is used to average the values in a neighbourhood of the cell.

Table 2. Classification Method Experimented in this Study

\begin{tabular}{|c|c|c|c|c|}
\hline \multirow{2}{*}{$\begin{array}{l}\text { Parameter } \\
\text { Calculation }\end{array}$} & \multirow[b]{2}{*}{ Author } & \multirow[b]{2}{*}{ Parameters } & \multicolumn{2}{|c|}{ Method } \\
\hline & & & Clustering & $\begin{array}{l}\text { Nested-Means } \\
\text { Divided } \\
\text { Parameters } \\
\end{array}$ \\
\hline $\begin{array}{l}\text { Single-cell } \\
\text { Analysis }\end{array}$ & Evans [7] & $\begin{array}{l}\text {-Slope gradient } \\
\text {-Aspect } \\
\text {-Plan curvature } \\
\text {-Profile curvature }\end{array}$ & 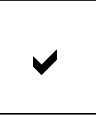 & \\
\hline & $\begin{array}{l}\text { Pike-Iwahashi } \\
\text { [6] }\end{array}$ & $\begin{array}{l}\text {-Slope gradient } \\
\text {-Texture } \\
\text {-Convexity }\end{array}$ & $\checkmark$ & $\checkmark$ \\
\hline $\begin{array}{l}\text { Multi-focal } \\
\text { Analysis }\end{array}$ & Pike [8] & $\begin{array}{l}\text {-Mean } \\
\cdot \text {-S.D. } \\
\cdot \text {-Variation coefficient } \\
\text { •Symmetry }\end{array}$ & $V$ & \\
\hline
\end{tabular}

The [6] classifies earth topography from DEMs by an unsupervised nested-means algorithm and a three-part geometric signature. It has been applied on the territory of the Province of Rome. The authors consider three morphometric parameters, in particular slope gradient, local convexity and surface texture, treating topography as a continuous random surface, independent of any spatial or morphologic orderliness imposed by fluvial activity or other geomorphic processes. The first parameter is computed by a single-cell analysis, while the others result from a multi-focal analysis, averaged on a 10-cell-radius circle.

This method identifies topographic types by using rules within a simple decision tree to apply image-processing operations to digital maps of the three variables. 


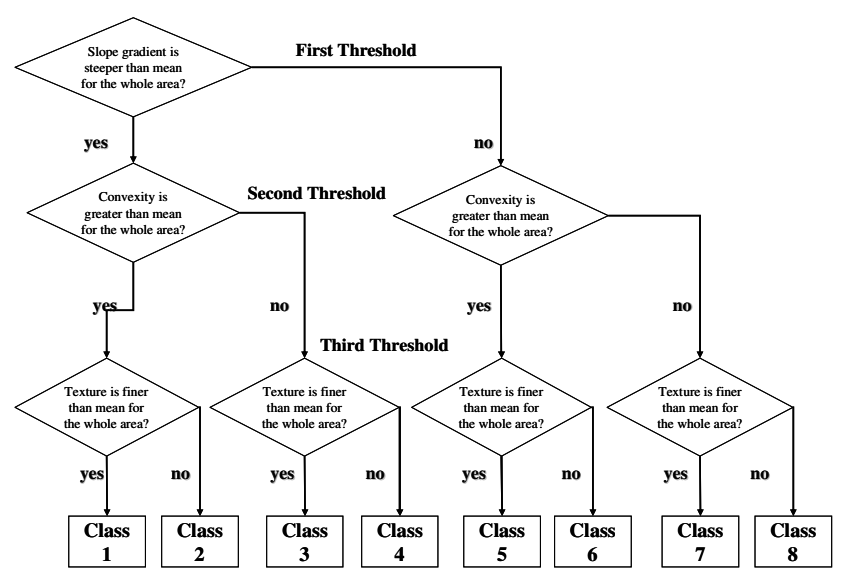

Fig. 1. Flow Chart for Automated Nested-Means Classification of Topography

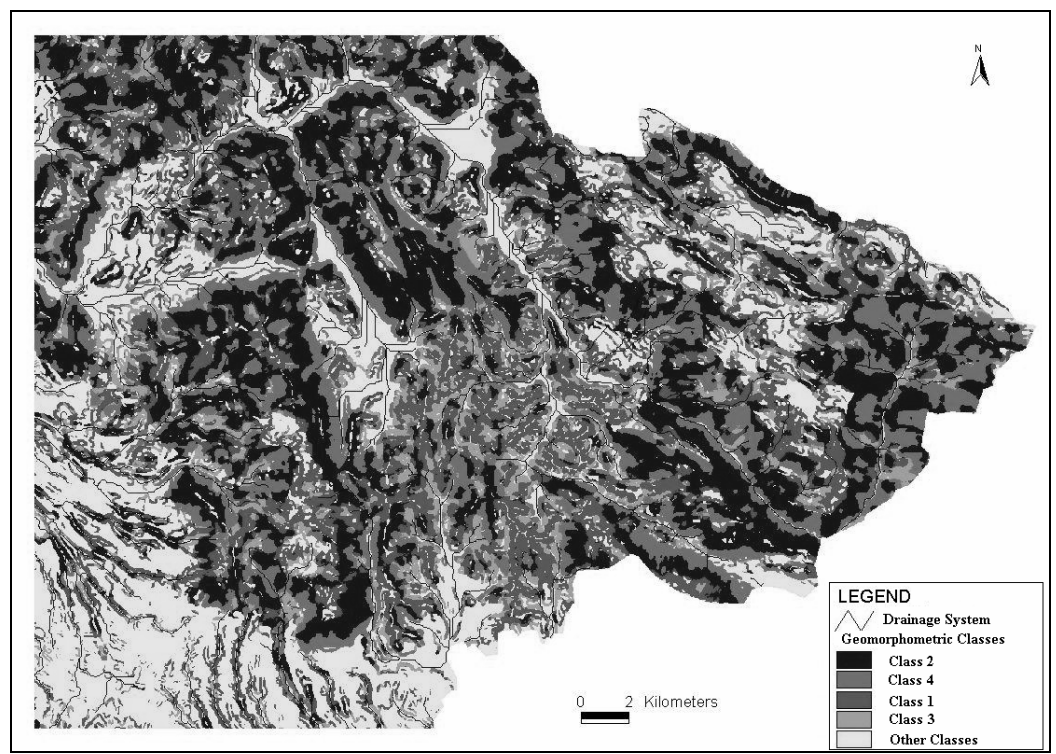

Fig. 2. Classes 1 to 4 of the Classification Obtained from [6]

The distribution of the three parameters is twofold-partitioned and the dividing threshold for each variable is its mean value, as represented in Figure 1. The result of the classification underlines a remarkable distinction among mountainside surfaces in four different classes, characterized by increasing values of elevation and slope gradient from Class 1 to 4 (Figure 2). The four classes from 5 to 8 represent earth surfaces less steeper than mean value, i.e. flat and semi-flat surfaces. This distinction (Classes 1 to 4 ) is fundamental to differentiate landslide correlation analysis results. 
The second group of experimented classification methods works by a statistical multivariate approach. The statistical multivariate analysis consists of a sequence of operations as represented in Figure 3. This semi-automated classification method classifies the analyzed territory by the principle of maximum internal homogeneity and minimum external homogeneity (cluster method). The mean of the variable distributions and the correlation between the distributions are the discriminating values in defining the eight classes.

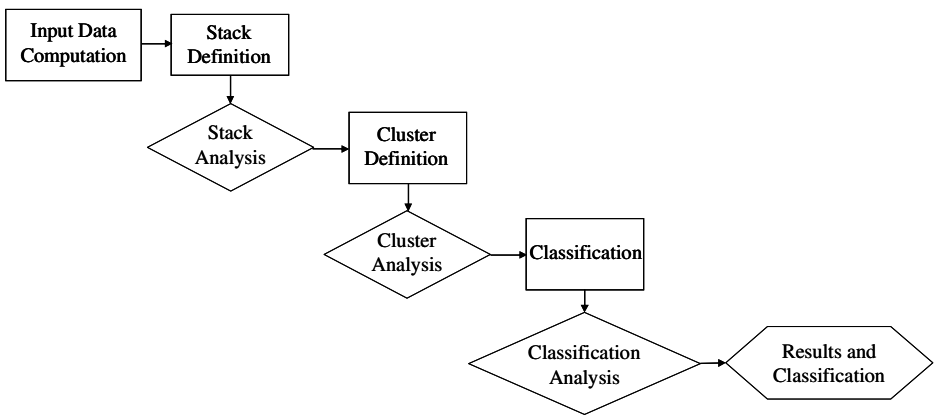

Fig. 3. Flow Chart for a Statistical Multivariate Analysis (Clustering)

First, the same parameters of [6] are considered as input data for this experimentation. The obtained results show a positive distinction among mountainside surfaces, but they do not identify important elements in trigger phenomena of landslides, such as hydrological ones. The eight classes show an increasing of elevation and slope gradient from class 1 to 8 .

The second experimented statistical multivariate study [7] considers five morphometric parameters, computed by a single-cell approach, such as altitude, slope gradient [10], aspect, profile convexity and plan convexity.

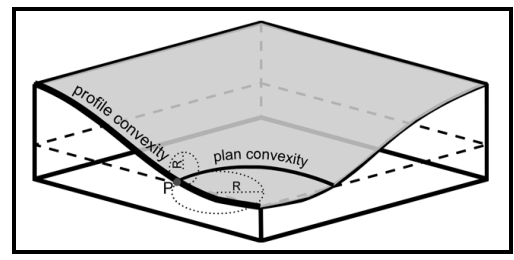

Fig. 4. Representation of Profile and Plan Convexity

The best result of multiple experimentations of this method is registered with slope gradient, aspect, profile curvature and plan curvature, normalized as follow:

$$
\bar{X}=\frac{x_{i}-\mu_{x}}{\sigma_{x}}
$$




\section{$\mu_{x}: \quad$ mean of $\mathrm{i}$-variable distribution \\ $\sigma_{x}: \quad$ standard deviation of i-variable distribution}

A particular of the most relevant classes obtained applying the previous method are represented in Figure 5.

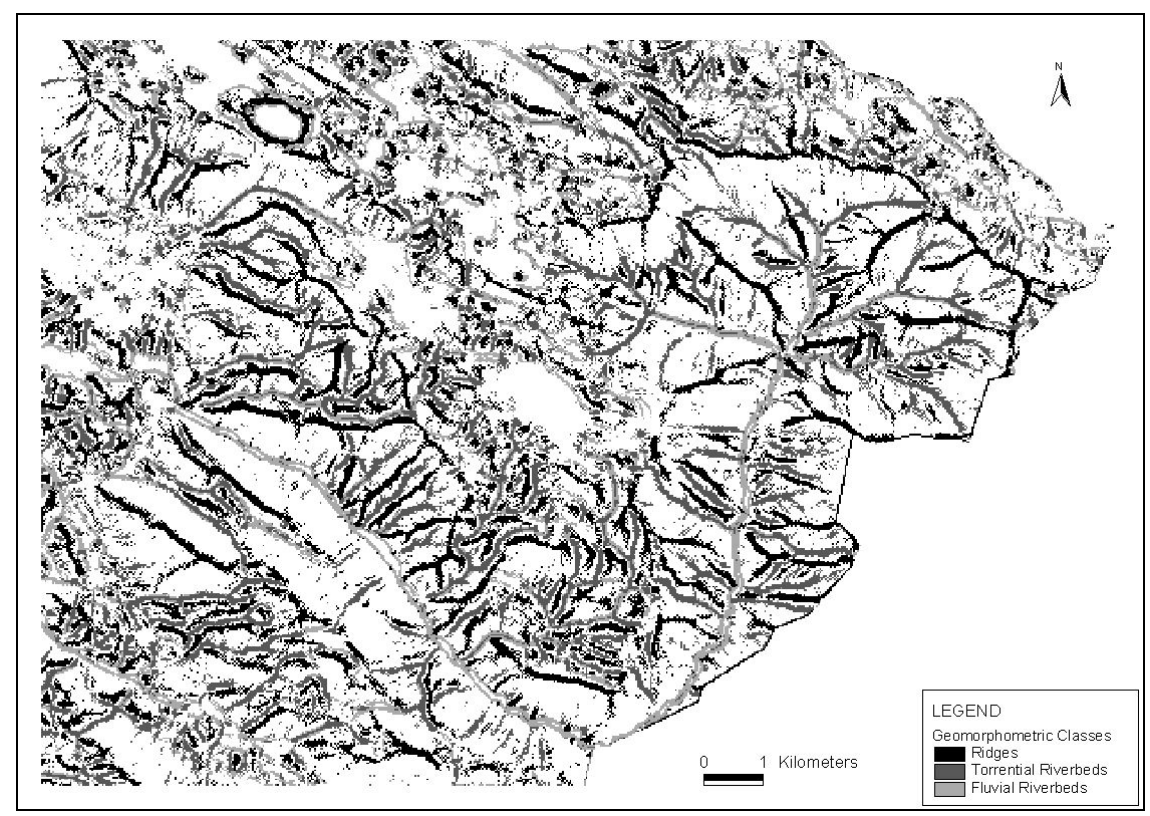

Fig. 5. A Particular of the Classification Obtained from [7]

The result of this method of classification underlines a remarkable distinction among terrain elements originated by hydrological and wind erosive activities, such as torrential (Class 1) and fluvial (Class 6) riverbeds and ridges (Class 8).

Among the considered morphometric variables, both curvatures discriminate these elements. In particular high values of both curvatures are registered in Class 8: there are high positive values of plan curvature corresponding to a convex surface and high negative values of section curvature corresponding to a concave surface. Both Class 1 and 6 have similar negative values of plan curvature (convergent water flow), but they differ in elevation and slope values: the first class has higher values of both variables than the second one. Furthermore, a topological continuity between classes 1 and 6 , representing torrential and fluvial riverbeds respectively, is detectable in Figure 5.

These elements are the main actors in triggering phenomena of landslides, while a distinction among different types of mountainside surfaces is essential in a landslide susceptibility analysis.

The experimented statistical multivariate classification method does not detect this distinction, creating only a class with all mountainside surfaces. 
A third set of derived parameters [8] are computed and considered as input data for the fourth experimentation. This method classifies the earth surface by a multivariate statistical approach, in the same way as the previously presented, but it considers statistical derived parameters, such as the mean, the standard deviation and variation and symmetry coefficients of the not-derived parameters. These statistical parameters are computed using ARC/INFO focal functions with different analysis windows according to the "topographic grain" concept. The result of this classification are not remarkable in discriminating different topographic classes of the analyzed territory.

\subsection{Classification Scheme}

The previous classification methods do not allow to reach the aim of the first step of analysis, i.e. a classification discriminating elements of territory relevant to a landslide susceptibility assessment. In this analysis, both terrain elements originated by hydrological and wind erosive activities, such as torrential and fluvial riverbeds and ridges, considered as main actors in trigger phenomena of landslides, and a distinction among mountainside surfaces in different classes are key factors.

Analyzing and comparing the different results obtained from previous classification methods, it is detectable that the multivariate statistical classification method considering not-derived parameters and the unsupervised nested-means procedure give the most interesting results.

Table 3. Comparison Among Different Obtained Results

\begin{tabular}{|c|c|c|c|c|}
\hline Method of Analysis & $\begin{array}{l}\text { Nested-means } \\
\text { Algorithm [6] }\end{array}$ & $\begin{array}{c}\text { Statistical } \\
\text { Multivariate }\end{array}$ & $\begin{array}{c}\text { Statistical } \\
\text { Multivariate [7] }\end{array}$ & $\begin{array}{c}\text { Statistical } \\
\text { Multivariate [8] }\end{array}$ \\
\hline Parameters & $\begin{array}{l}\text { Slope Gradient } \\
\text { Texture } \\
\text { Local Convexity }\end{array}$ & $\begin{array}{l}\text { Slope Gradient } \\
\text { Texture } \\
\text { Local Convexity }\end{array}$ & $\begin{array}{l}\text { Slope Gradient } \\
\text { Aspect } \\
\text { Plan Curvature } \\
\text { Profile Curvature }\end{array}$ & $\begin{array}{l}\text { Mean } \\
\text { Standard Deviation } \\
\text { Symmetry } \\
\text { Variation Coefficient }\end{array}$ \\
\hline Classification & Good & Good & Good & Unacceptable \\
\hline $\begin{array}{c}\text { Mountainside Surfaces } \\
\text { Distinction }\end{array}$ & Best & Good & Good & Unacceptable \\
\hline $\begin{array}{c}\text { Trigger Elements } \\
\text { Distinction }\end{array}$ & Good & Unacceptable & Best & Unacceptable \\
\hline
\end{tabular}

The statistical multivariate analysis considering DEM-first-derived parameters, such as slope gradient, aspect and both curvatures, returns a remarkable distinction among landslide trigger elements (Classes 1, 6, 8), but also a class (n.7) that contains all mountainside surfaces. The second classification method allocates these surfaces into four different classes. From a comparison between these classes (from 1 to 4 ) and Class 7 of the first classification method, it is detectable that the four classes essentially coincide with Class 7; analyzing areas of these classes it is well-rendered that the four classes, including all grid-cells with more than 7.5 slope degree (mean of the distribution), cover a larger area than Class 7 and therefore in general they always comprise it, but they have a more specific division of mountainside surfaces.

Thus, the development of a new classification method that integrates the positive results of the experimented procedures and avoids negative ones is necessary to correctly analyze landslide susceptibility. 


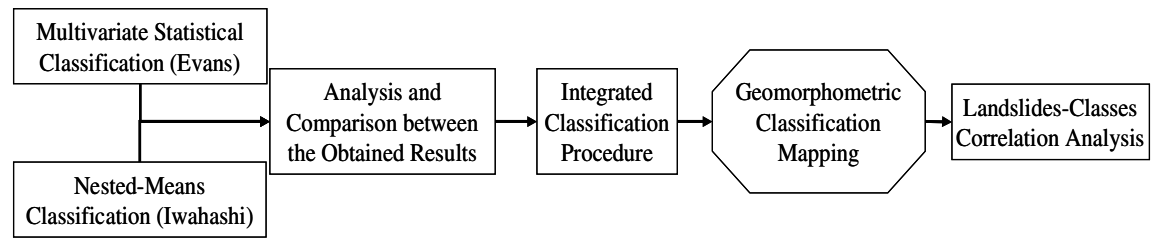

Fig. 6. Flow Chart of the Integrated Classification

The integrated classification is developed on a grid-cell based analysis. Both classifications are 30x30m square-grid data, like the input DEM. The output grid have eleven different groups of cells and the selection of relevant classes of the input classifications is implemented by a conditional function (If...Then...), as represented in Figure 7.

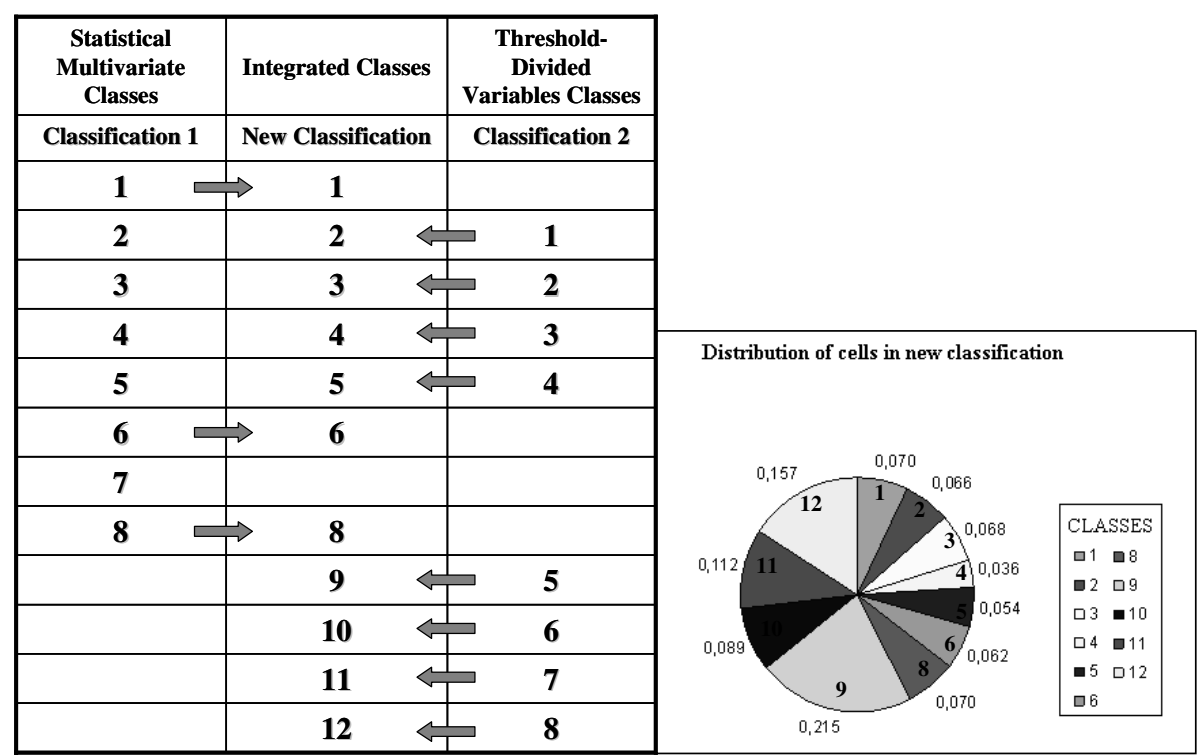

Fig. 7. Representation of the Procedure Adopted in Defining the New Classification Method from the Two Previous Ones and Percentage Distribution of Cells within the New Classes

The relevant classes of the first classification, i.e. terrain elements originated by hydrological and wind erosive activities, such as torrential (Class 1) and fluvial (Class 6 ) riverbeds and ridges (Class 8) remain unchanged. All classes of the second classification are integrated in the new one.

Therefore, in this classification Classes $(2-5)$ represent mountainside surfaces, now distinguished in four classes and previously all included in Class 7 (Classification 1). Analyzing morphological values of these four classes (Table 4), it is easy detectable an increasing of elevation and slope gradient mean in the order Class 4 , 
Table 4. Elevation and Slope Value Distributions in the Obtained Classes

\begin{tabular}{|c|c|c|c|c|c|c|c|c|c|c|}
\hline \multirow{2}{*}{ CLASS } & \multicolumn{5}{|c|}{ DEM } & \multicolumn{5}{|c|}{ SLOPE } \\
\cline { 2 - 12 } & MIN & MAX & RANGE & MEAN & MEAN_ST & MIN & MAX & RANGE & MEAN & MEAN_ST \\
\hline $\mathbf{1}$ & 0 & 1815,4 & 1817,4 & 430,7 & 906,7 & 0 & 44,0 & 44,0 & 12,5 & 22,0 \\
\hline $\mathbf{2}$ & 0 & 1839,7 & 1848,7 & 368,5 & 915,4 & 7,5 & 41,8 & 34,3 & 13,2 & 24,6 \\
\hline $\mathbf{3}$ & 4,9 & 1828,9 & 1824,0 & 613,4 & 916,9 & 7,5 & 46,0 & 38,5 & 20,1 & 26,7 \\
\hline $\mathbf{4}$ & 4,3 & 1770,8 & 1766,4 & 310,6 & 887,6 & 7,5 & 37,0 & 29,5 & 12,7 & 22,3 \\
\hline $\mathbf{5}$ & 4,0 & 1719,9 & 1715,8 & 477,6 & 861,9 & 7,5 & 45,4 & 37,9 & 17,7 & 26,5 \\
\hline $\mathbf{6}$ & 0 & 1815,9 & 1829,8 & 297,5 & 908 & 0 & 45,2 & 45,1 & 9,4 & 22,6 \\
\hline $\mathbf{8}$ & 0 & 1846,3 & 1852,1 & 494,0 & 920,2 & 0 & 52,1 & 52,1 & 14,0 & 26,0 \\
\hline $\mathbf{9}$ & 0 & 1821,8 & 1828,6 & 165,4 & 907,5 & 0 & 7,5 & 7,5 & 3,2 & 3,7 \\
\hline $\mathbf{1 0}$ & 0 & 1820,2 & 1823,4 & 114,5 & 908,5 & 0 & 7,5 & 7,5 & 2,0 & 3,7 \\
\hline $\mathbf{1 1}$ & 0 & 1732,4 & 1734,9 & 168,5 & 864,9 & 0 & 7,5 & 7,5 & 3,0 & 3,7 \\
\hline $\mathbf{1 2}$ & 0 & 1673,8 & 1676,2 & 118,4 & 835,7 & 0 & 7,5 & 7,5 & 1,3 & 3,7 \\
\hline
\end{tabular}

Class 2, Class 5 and finally Class 3. Physically, this succession correctly corresponds to the natural trend of mountainside surfaces.

Classes from 9 to 12 represent flat or semi-flat surfaces, introducing a new hydrological feature. In fact, comparing the classification with the drainage system of this territory it is clearly visible the overlap with Class 11, i.e. fluvial riverbeds located in valley floor. These further elements complete the characterization of the hydrological feature, filling the registered gap of the first classification.

Therefore, the first step of analysis is complete and the obtained results allow to analyze the correlation between the identified classes and landslides.

\section{Landslide Susceptibility Assessment}

Assessing the correlation levels between topographic signatures and landslide events is the second objective of this work. Its final product, a first level of a landslide susceptibility map, provides Civil Protection planners with a practical and cost-effective way to zone areas potentially susceptible to landslide occurrences.

Interpretation of future landslide occurrence requires an understanding of conditions and processes controlling landslides in the study area [11]. Two physical factors, past history and slope steepness, are the minimum components necessary to assess landslide susceptibility. It is also desirable to add a hydrologic factor to reflect the important role which ground water often plays in the occurrence of landslides.

As explained in Chapter 3.2, the final result of the first step of analysis is a terrain classification which considers the shapes of both physical and hydrological features and therefore it could be optimally used as input data in a landslide susceptibility assessment. 
The overlap of the Landslide Inventory Map with the integrated classification allows to identify which classes are associated with a certain type of landslide and which are not. To assess different levels of correlation it is used a statistical approach.

$$
C_{k j}=\frac{\sum_{i=1}^{m} \text { Area }_{i}}{\sum_{i=1}^{n} \text { Area }_{i}}
$$

$\mathrm{k}: \quad$ type of landslide

j: $\quad$ geomorphometric class

$\mathrm{m}$ : number of $\mathrm{k}$-type landslides in class $\mathrm{j}$

$\mathrm{n}$ : total number of k-type landslides

The correlation values are computed in (2) for each geomorphometric class in relation to each registered type of landslide.

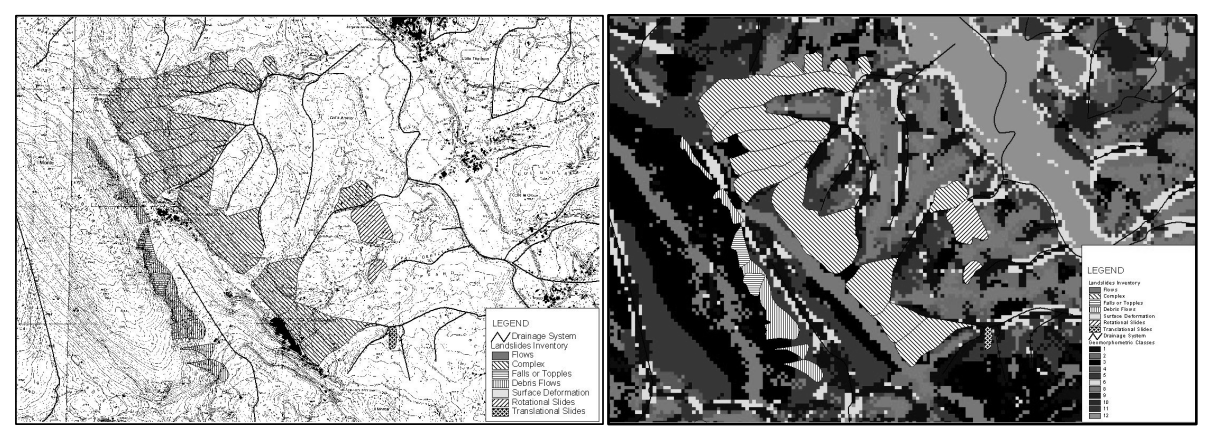

Fig. 8. Landslides Inventory Map with Drainage System and Overlapping of the LIM with the Geomorphologic Classes

The Landslide Inventory differentiates seven types of phenomena [12], such as falls or topples (Type 2), translational and rotational slides (Types 4 and 5), flow (Type 1), debris flow (Type 6), surface deformation (Type 3) and complex (Type 7).

The results of the statistical correlation analysis, presented in Figure 9, show a remarkable distinction among classes representing mountainside surfaces and high percentage of correlation only for a subset of these classes. In particular only five classes are correlated, in a significant way, to landslide phenomenon; they cover the signatures mainly relevant for landslide occurrence, such as torrential and fluvial riverbeds (Classes 6 and 1), main actors in trigger phenomena of landslides.

These results, from the Civil Protection point of view, mean that the percentage of territory to be investigated with a more specific predictive analysis is reduced. 


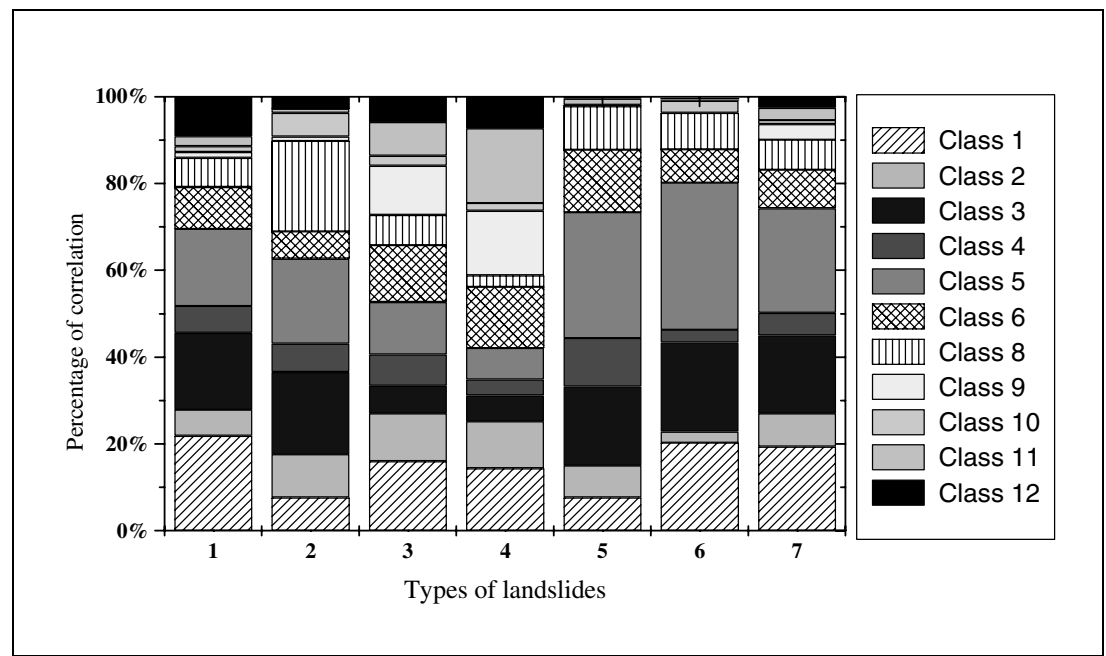

Fig. 9. Schematic Representation of Percentage Values of Correlation between Each Type of Landslide and Geomorphometric Class

Analyzing single class correlation values, we note that:

- Class 8 (ridges) presents an high correlation value with "falls or topples" landslide type;

- Class 1 (torrential riverbeds) is one of the most correlated identified class, in particular in relation with various types of flow (debris, earth/rock flow). A low correlation percentage results with "falls or topples";

- Class 3 (high mountainside surfaces) presents high values for all types of landslide, excepted for surface deformations and translational slides. It presents the highest value for debris flows;

- Class 5 (medium mountainside surfaces) is the most correlated class; it presents values higher than $30 \%$ for rotational slides and debris flows, values between $10 \%$ and $20 \%$ for "flows, falls or topples and surface deformations", values lower than $10 \%$ only for translational slides;

- Class 6 (fluvial riverbeds) is correlated in particular with the two types of slides and surface deformations.

Analyzing the obtained distinction among mountainside surfaces in the four identified classes and their correlation values with landslides, it is possible to exclude Classes 2 and 4 from group characterized by high correlation levels. At the same time, it is clearly detectable that differences exist also between Class 3 and 5: in particular Class 5 , covering only the $5.4 \%$ of the investigated territory, is characterized by high values of correlation. The final result is the identification of a more precise definition of the limits of these zones and which types of events affect every class, especially for those classes with higher susceptibility levels. 


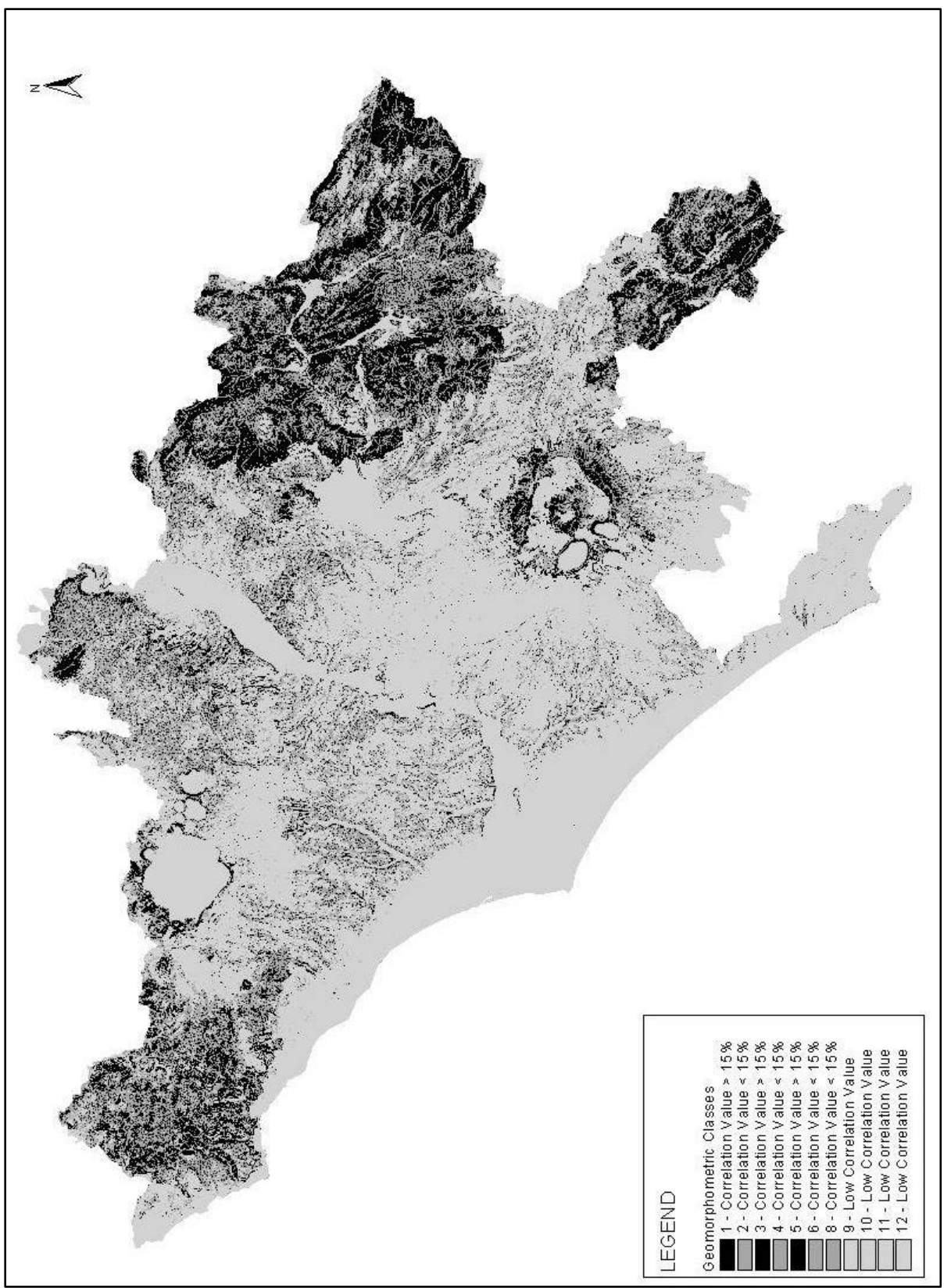

Fig. 10. Representation of the Geomorphometric Classes by Correlation Values with Landslides 


\section{Conclusions}

A reliable semi-automated method, working by a geomorphometric approach, is applied to the territory of the Province of Rome in order to define a classification oriented to landslide susceptibility assessment. The main objective is reached by means of intermediate steps.

The result of the first step of analysis is the individuation of six parameters, slope gradient, aspect, plan and section curvature, local convexity and surface texture, as a set of measures, a geometric signature, that describes topographic form to distinguish among different landscapes; these parameters are computed from a 30x30m squaregrid digital elevation model (DEM). The result of the second step of analysis is the classification of the analyzed territory in eleven classes using the geometric signature analytic tool. The results of the classification method have been validated by means of a GIS-based comparison and analysis between the different obtained geomorphometric classes and the theme of the drainage system of this territory and an analytic investigation of the elevation, slope, plan and section curvatures values distributions in each class. Finally, the eleven classes are statistically correlated with the Landslides Inventory of the analyzed territory.

This work represents a useful tool in a landslide susceptibility analysis, due to its capacity to discriminate, in a large-scale context, and by simply using a DEM as input data, the specific part of a territory more meaningful for the landslide occurrences. Of course, it provides just a first level of a susceptibility assessment, but the application of this repeatable and reliable procedure may return the best results in a short time and with low economic resources, providing specific useful information in planning greatscale Civil Protection investigations and operations.

However, we have to consider that to carry out a complete susceptibility assessment is necessary the integration of these results with information concerning the physical factors influencing landslide occurrences. Finally, in order to determine the extent and the strength of landslide hazard it is necessary to identify those areas which could be affected by a damaging landslide and to assess the probability of the landslide occurrence within some time period. In general, the evaluation of a time frame for the occurrence of a landslide is not simple to determine even under ideal conditions.

Factors influencing where landslides occur, can be divided into two groups, permanent and variable. Permanent factors represent the landscape which remains unchanged or varies little from a human perspective. The steepness of a slope, for example, presents changes only with the passage of long periods of time. By examining existing landslides in an area, it is possible to recognize how permanent factors contributed to these slope failures. Identifying conditions and processes promoting past instability makes it possible to use these factors to estimate landslide susceptibility. Variable factors are terrain characteristics that change quickly as a result of some triggering event. Ground vibrations due to earthquakes, a rapid rise in groundwater levels, and increased soil moisture due to intense precipitation are examples of variable factors.

In order to evaluate the probability of the landslide occurrence, and subsequently the landslide hazard, it is necessary to integrate the results obtained in this study with other physical factors, then to assess the probability of the occurrence of these triggering variable factors and finally to integrate this assessment with landslide susceptibility analysis. These analysis will be subjects of future studies. 


\section{References}

1. Carrara, A., Cardinali, M., Guzzetti, F., Reichenbach, P.: GIS-Based Techniques for Mapping Landslide Hazard

2. Enzmann, R.D.: Introduction to the Section on Signatures, in Planetology and Space Mission Planning. Ann. New York Acad.Sci. 140 (1966)

3. Pike, R.J.: The Geometric Signature: Quantifying Landslide-Terrain Types from Digital Elevation Models, Mathematical Geology (1988)

4. Pike, R.J.: Geometric Signatures of Intermediate-Scale Topography. Geol. Soc. Amer. Abst. Prog. 18 (1986)

5. Hansen, A.: Landslide Hazard Analysis. In: Brunsden, D., Prior, D.B. (eds.) Slope instability, pp. 523-602. Wiley, New York (1984)

6. Iwahashi, J., Pike, R.J.: Automated classifications of topography from DEMs by an unsupervised nested-means algorithm and a three-part geometric signature, Geomorphology (2006)

7. Evans, I.S.: General Geomorphometry. In: Goudie, A.S. (ed.) Geomorphological Techniques (1981)

8. Pike, R.J.: Elevation Relief Ratio, Hypsometric Integral, and Geomorphic Area-Altitude Analysis. In: Geological Society of American Bulletin (1971)

9. Prima, O.D.A., Echigo, A., Yokoyama, R., Yoshida, T.: Supervised Landform Classification of the Northeast Honshu from DEM-derived Thematic Map. Geomorphology 78 (2006)

10. Evans, I.S.: An Integrated System of Terrain Analysis and Slope Mapping, University of Durham, England (1979)

11. Department of Regional Development and Environment Executive Secretariat for Economic and Social Affairs Organization of American States with support from the Office of Foreign Disaster Assistance United States Agency for International Development: Primer on Natural Hazard Management in Integrated Regional Development Planning (1991)

12. Varnes, D.J.: Landslide: Analysis and Control, Transportation R.B.N.A.S. Washington, vol. $176(1978)$ 